\title{
ERRATUM
}

doi:10.1038/nature08546

\section{El Niño in a changing climate}

Sang-Wook Yeh, Jong-Seong Kug, Boris Dewitte, Min-Ho Kwon, Ben P. Kirtman \& Fei-Fei Jin

Nature 461, 511-514 (2009)

In Figure 4 of this letter, the key for the 20C3M ensemble (red line) and the SRESA1B ensemble (blue line) were inadvertently mislabelled. The correct figure is shown below.

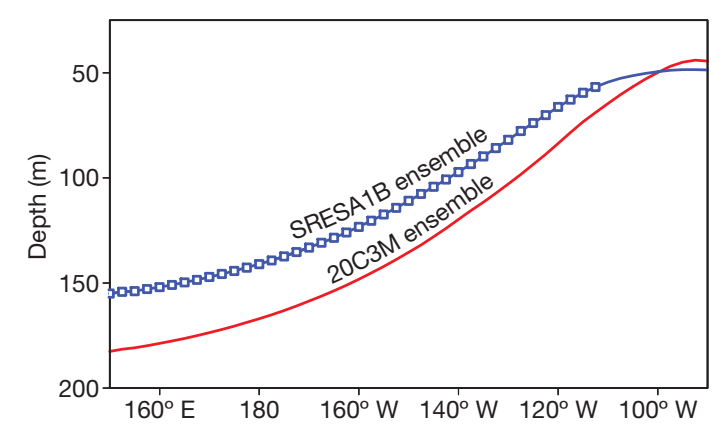

